Rev. Saúde públ., S. Paulo, 25(4): 315-7, 1991

\title{
Epidemia de dengue em Ribeirão Preto, SP, Brasil: Nota prévia
}

\author{
Dengue in the Southeast Region of Brazil: a preliminary note
}

Ricardo José Soares Pontes*, Amaury Lelis Dal Fabbro**, Gutemberg de Melo Rocha*,
Roseli Claudino Santiago***, Luis Tadeu M. Figueiredo*, Ana Alice M.C. Castro e Silva***,
Vilma Delphino de Oliveira Garotti****, Josely Mendonça Pereira Pintyán

\begin{abstract}
Pontes, R.J.S. et al. Epidemia de dengue em Ribeirão Preto, SP - Brasil: Nota prévia. Rev. Saúde públ., S. Paulo, 25: 315-7, 1991. Apresenta-se Nota preliminar de uma epidemia de dengue, ocorrida em Ribeirão Preto, São Paulo, Brasil, no período de novembro de 1990 a março de 1991. Descreve-se a ocorrência de um caso de "Dengue Hemorrhagic Fever/Dengue Shock Syndrome" Grau II (DHF/DSS) e de dois óbitos associados ao dengue devido a síndrome de choque. Alerta clínicos e epidemiologistas quanto à possibilidade de ocorrência de casos isolados de DHF/DSS durante epidemias de dengue clássico em áreas antes indenes à doença.
\end{abstract}

Descritores: Dengue, epidemiologia. Surtos de doenças.

\section{Introdução}

Em novembro de 1990 ocorreu uma epidemia de dengue no Município de Ribeirão Preto, Estado de São Paulo, Brasil. Na presente comunicação faz-se uma descrição preliminar de alguns aspectos de importância epidemiológica observados durante 0 processo epidêmico e que merecem divulgação imediata, principalmente pela ocorrência de dois óbitos associados ao dengue. O estudo mais detathado e aprofundado da epidemia será publicado posteriormente.

\section{A Epidemia}

O processo epidêmico******* foi detectado pelos serviços de saúde pública na segunda quinzena de novembro de 1990 (174 casos notificados) em um bairro periférico de Ribeirão Preto, provavel-

\footnotetext{
* Faculdade de Medicina de Ribeirão Preto da Universidade de São Paulo

** Superintendência de Controle de Endemias de Ribeirão Preto, SP

*** Secretaria Municipal de Saúde de Ribeirão Preto, SP

**** Escritório Regional de Saúde de Ribeirão Preto, SP

***** Instituto Adolfo Lutz de Ribeirāo Preto, SP

****** Hospital das Clínicas da Faculdade de Medicina de Ribeirão Preto da Universidade São Paulo

******* Fonte de informaçס̄es: Fichas de notificação de casos de dengue. Vigilância Epidemiológica. Secretaria Municipal de Saúde, Ribeirẩo Preto.
}

Separatas/Reprints: R.J.S. Pontes - Av. Bandeirantes, 3900 14049 Ribeirão Preto, SP - Brasil.

Publicação financiada pela FAPESP. Processo 90/4602-1. mente quando já se achava em franca ascensão. Rapidamente houve o aumento da incidência, com expansão da epidemia para vários bairros da cidade, atingindo-se o pico máximo no mês de dezembro ( 7.325 casos notificados). Progressivamente observou-se o declínio das notificaçoes nos meses de janeiro, fevereiro e março $(1.122,277$ e 65 , respectivamente), quando as autoridades de saúde anunciaram oficialmente o fim da epidemia. As amostras de sangue examinadas mostraram a circulação apenas do vírus Den-1.

As medidas de controle constaram de: a- nebulização UBV (ultra baixo volume), utilizando-se máquinas pulverizadoras acopladas a viaturas, num total de onze ciclos com inseticidas piretróides; $b$ - tratamento larvicida focal e perifocal com inseticidas organofosforados nos bairros com maior transmissão; foi realizado simultaneamente nesses locais pulverização UBV intradomiciliar com equipamentos portáteis; c- arrastão de limpeza com retirada e eliminação de recipientes criadouros de Aedes aegypti em toda área urbana do município; d- intensa mobilização da opinião pública pelos meios de comunicação de massa e educação sanitária por parte dos serviços de saúde.

A Figura apresenta a evolução dos casos notificados no período de novembro de 1990 a março de 1991, mostrando a correspondência entre a curva declinante do índice de Breteau (novembro: 6,9; janeiro: 2,1 ; fevereiro: 0,3 ; março: 1,6 ) com a tendência decrescente da curva epidêmica. A análise do impacto das medidas de controle em relação ao declínio da epidemia será objeto de discussão em trabalho posterior. 


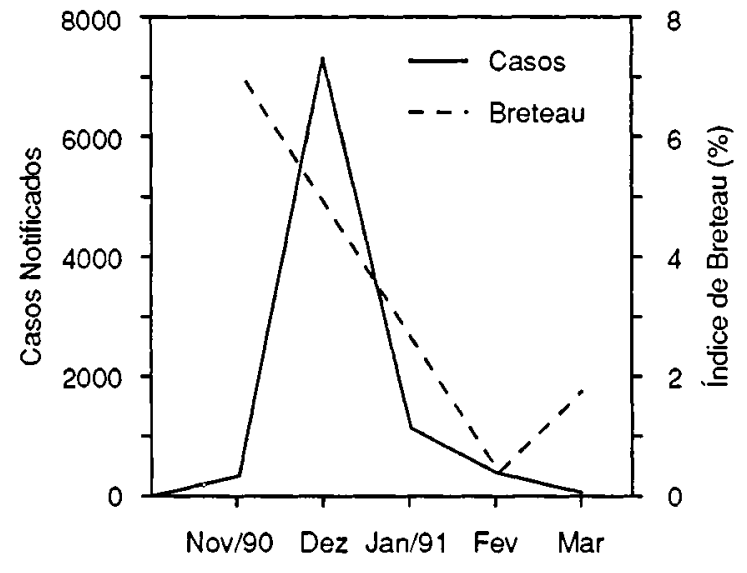

Figura - Casos notificados de Dengue e índice de Breteau, em Ribeirāo Preto - Novembro de 1990 a Março de 1991.

Torna-se relevante em termos epidemiológicos chamar a atenção para o fato de que, mesmo depois das intensas medidas de controle postas em prática, continuaram a ser notificados casos isolados com confirmação laboratorial em diversos bairros, sugerindo que o processo parece se tornar endêmico no município. A persistência de casos residuais mostra a possibilidade de manter-se a transmissão da doença mesmo com indices de Breteau muito baixos (abaixo dos 5\% normalmente utilizados como limiar de risco epidêmico), provavelmente às custas de áreas na cidade onde a infestação era focalmente mais elevada.

\section{Febre do Dengue Hemorrágico (DHF/DSS) e Óbitos Associados ao Dengue}

Durante a epidemia foram observados dois óbitos em adultos associados ao dengue e um caso compatível com DHF/DSS Grau II, de acordo com a classificação da $\mathrm{OMS}^{4}$.

Ambos os pacientes que foram a óbito apresentaram evidências clínicas, antecedentes epidcmiológicos e dados laboratoriais compatíveis com quadro de dengue, ocorrendo o choque circulatório no curso da efermidade febril aguda. Não se observou fenômenos hemorrágicos clínicos ou nos achados de autópsia. A investigação epidemiológica e a autópsia não mostraram qualquer doença de base ou fator associado que justificasse o quadro apresentado.

A literatura refere a ocorrência de casos isolados de DHF/DSS durante epidemias de dengue clássico em pacientes primo-infectados, mostrando também variações no espectro clínico de apresentação daquela enfermidade ${ }^{1,3,5}$. Entretanto, devido a ampla divulgação da Teoria Imunopatológica de Halstead ${ }^{2,6} \mathrm{e}$ a inexistência entre nós de experiência clínica anterior no manuseio dessas situações, nenhum dos dois casos de óbitos asssociados ao dengue tiveram incluídas entre as hipóteses diagnósticas a possibilidade de DHF/DSS. Nessa direção, um dos pacientes com quadro típico de dengue clássico apresentou piora pelo quarto dia da enfermidade, tendo procurado diversos serviços de saúde sem que fosse deduzida a possibilidade de DHF/DSS, até que deu entrada no Hospital das Clínicas de Ribeirão Preto, com choque irreversivel.

Embora 0 estudo detalhado dos citados episódios seja objeto de publicação posterior, consideramos importante e oportuno sua divulgação imediata com o objetivo de alertar os epidemiologistas e clínicos da possibilidade de tais ocorrências. A manter-se o quadro epidemiológico atual, epidemias de dengue continuarão a acontecer em áreas indenes à doença. Nessas situações, por tratar-se de um fenômeno raro e pouco divulgado entre nós, os casos esporádicos de DHF/DSS dentro de uma epidemia de dengue clássico poderão não ser identificados com a precocidade necessária para, com manejo adequado, interromper-se uma evolução desfavorável. É necessário que os serviços de saúde estejam atentos na vigilância, na orientação dos pacientes com dengue e no diagnóstico precoce dessas situações de modo a não permitir a ocorrência de óbitos que eventualmente poderiam ser evitados.

\section{Agradecimentos}

À Equipe de Vigilância Epidemiológica: Maria Luiza S. S. Maria, Iara C. Ungari, Aparecida H. C. Ferreira, Sueli C. Gera, Daniel A. Capuano, Tany M. S. Biondi, Angela M. Serafim, Luiza S. S. Aliberti, Maria Teresa L. M. Reis, Esmeralda T. S. Henriques, Zélia de Freitas, Lairce Aparecida Tibério, pela colaboração na coleta de dados.

PONTES, R.J.S. et al. [Dengue in the Southeast Region of Brazil: preliminary note.] Rev. Saúde públ., 25:315-7, 1991. A preliminary note on an outbreak of dengue which occurred in the city of Ribeirão Preto, State of S. Paulo, Brazil, from November 1990 to March 1991, is given. One case of Level II Dengue Hemorrhagic Fever/Dengue Shock Syndrome (DHF/DSS) and two deaths associated with dengue related shock are described. Clinicians and epidemiologists are alcrted to the possibility of sporadic DHF/DSS cases during outbreaks of classical dengue in formerly free areas.

Keywords: Dengue, epidemiology. Disease outbreaks. 


\section{Referências Bibliográficas}

1. BARNES, W. J. S. \& ROSEN, L. Fatal hemorrhagic disease and shock associated with primary dengue infection on a Pacific Island. Amer. J. trop. Med. Hyg., 23: 495-506, 1974.

2. HALSTEAD, S. The pathogenesis of dengue: molecular epidemiology in infectious disease. Amer. J. Epidem., 114: $632-48,1981$

3. LOPES-CORREA, R. H.; CLNNE, B. L. , RAMIREZRONDA, C.; BERMUDEZ, R.; SATHER, G. E.; KUNO, G. Dengue fever with hemorrhagic manifestations: a report of three cases from Puerto Rico. Amer. $J$. trop. Med. Hyg; 27: 1216-24, 1978.
4. ORGANIZAÇÃO MUNDIAL DA SAUDE. Dengue he morrágico: diagnóstico, tratamento e controle. Genebra, 1987

5. REED, D.; MAGUIRE, T.; MATAIKA, J. Type 1 dengue with hemorrhagic disease in Fiji: epidemiologic findings. Amer. J. trop. Med. Hyg, 26: 784-91, 1977.

6. SECRETARIA DE ESTADO DA SAUDE: Manual de vigilância epidemiológica-dengue. São Paulo, 1987.

Recebido para publicação em 25/06/1991. Aprovado para publicação em 12/07/1991. 